\title{
Clinicopathological and prognostic significance of mitogen- activated protein kinases (MAPK) in breast cancers
}

\author{
Dena A. J. Ahmad ${ }^{1,2} \cdot$ Ola H. Negm ${ }^{3,4} \cdot$ M. Layth Alabdullah ${ }^{5}$ Sameer Mirza ${ }^{6}$. \\ Mohamed R. Hamed $^{3,4} \cdot$ Vimla Band $^{6}$ - Andrew R. Green ${ }^{1}$ Ian O. Ellis ${ }^{1}$. \\ Emad A. Rakha ${ }^{1}$
}

Received: 24 August 2016/Accepted: 26 August 2016/Published online: 3 September 2016

(c) The Author(s) 2016. This article is published with open access at Springerlink.com

\begin{abstract}
Background Mitogen-activated protein kinases (MAPKs) are signalling transduction molecules that have different functions and diverse behaviour in cancer. In breast cancer, MAPK is related to oestrogen receptor (ER) and HER2. Methods Protein expression of a large panel of MAPKs (JNK1/2, ERK, p38, C-JUN and ATF2 including phosphorylated forms) were assessed immunohistochemically in a large $(n=1400)$ and well-characterised breast cancer series prepared as tissue microarray. Moreover, reverse phase protein array was applied to quantify protein
\end{abstract}

Electronic supplementary material The online version of this article (doi:10.1007/s10549-016-3967-9) contains supplementary material, which is available to authorized users.

Ola H. Negm-joint first author.

Ola H. Negm

ola.negm@nottingham.ac.uk

1 Division of Cancer and Stem Cells, Department of Histopathology, School of Medicine, Nottingham City Hospital, The University of Nottingham and Nottingham University Hospitals NHS Trust, Nottingham, UK

2 Department of Pathology, Mosul Medical School, University of Mosul, Mosul, Iraq

3 School of Medicine, Queen's Medical Hospital, University of Nottingham, Derby Road, Nottingham NG7 2UH, UK

4 Faculty of Medicine, Medical Microbiology and Immunology Department, Mansoura University, Mansoura, Egypt

5 Academic Unit of Clinical Oncology, School of Medicine, Nottingham City Hospital, University of Nottingham, Nottingham, UK

6 Department of Genetics, Cell Biology and Anatomy, University of Nebraska, Omaha, USA expression of MAPKs in six breast cancer cell lines with different phenotypes including HER2-transfected cells. Results MAPKs expression was associated with clinicopathological variables characteristic of good prognosis. These associations were most significant in the whole series and in the ER+ subgroup compared to other BC classes. Most of MAPKs showed a positive association with ER, BCL2 and better outcome and were negatively associated with the proliferation marker Ki67 and p53. Association of MAPK with HER2 was mainly seen in the ER- subgroup. Reverse phase protein array confirmed immunohistochemistry results and revealed differential expression of MAPK proteins in ER+ and ER - cell lines. Conclusions MAPKs are associated with good prognosis and their expression is mainly related to ER. Studying a large panel rather than individual biomarkers may provide improved understanding of the pathway.

Keywords MAPK signalling pathways - Reverse phase protein microarray (RPPA) $\cdot$ Breast cancer

\section{Introduction}

Mitogen-activated protein kinases (MAPKs) are evolutionary conserved enzymes which function as signal transduction pathways that regulate fundamental cell activities including gene expression, cellular processes such as growth, proliferation, differentiation, migration and apoptosis. MAPKs' stimulation starts from stimulation of receptor tyrosine kinase (RTK) by growth factors, cytokines, heat shock, mitogen, osmotic stress or stress factors [1]. Stimulated receptors associate with certain adaptor proteins that in turn enhance the recruitment of guanine nucleotide exchange factors (GEFs) in the cell membrane. 
The latter can stimulate some small GTPase proteins $(\mathrm{H}-$ RAS, N-RAS and K-RAS) which eventually regulate the switch from GDP to GTP and vice versa [2]. GEFs can enhance the formation of RAS GTP which is the active form that can bind to different downstream proteins such as RAF, members of PI3K pathway and RAL-GEF-RAL cascade [3-5]. RAF proteins directly phosphorylate MEK1/2 [6, 7] and can activate ERK1/2 [6] which subsequently can enhance different factors such as transcription factors, kinases and phosphatases $[2,6]$.

Several groups of MAPK family have been identified, including extracellular regulatory kinase (ERK1/2/5, also known as classical MAPK), p38 MAPK and c-Jun N-terminal kinase/stress-activated protein kinases (JNK1/2/3/ SAPKs). Activation of each group requires dual phosphorylation of threonine and tyrosine within the activation loop of the MAPK through a three-tiered cascade composed of MAPK, MAPK kinases (MEKs) and MAPKKK. MEKs are specific for each isoform of MAPK family. For instance, ERK1/ERK2 is activated by MEK1 and MEK2, JNK is activated by MEK4 and MEK7 and p38 MAPK is activated by MEK3 and MEK6 [8, 9].

The function of MAPKs in breast cancer (BC) is complex due to different responses they modulate and their interaction with different pathways [9-11]. MAPKs have been investigated in $\mathrm{BC}$ including their interaction with oestrogen receptor (ER) and HER2; however, conflicting results were reported and the exact role of MAPKs in BC and their interaction with ER and HER2 remain to be determined [12-14].

The aim of this study is to investigate the role of MAPK signalling cascade in $\mathrm{BC}$ utilising a large panel of biomarker and a large well-characterised series of early stage BC prepared as TMA using IHC. MAPK expression was assessed in the different molecular classes based on expression of ER and HER2 status. In addition, reverse phase protein array (RPPA) was employed to quantify MAPK protein expression levels in different $\mathrm{BC}$ cell line phenotypes including the impact of HER2 transfection in the ER+ and ER - cell lines. This retrospective study adheres to REMARK criteria [15].

\section{Materials and methods}

In this study, 1400 cases of unselected operable invasive BC series were included; these are part of the Nottingham Tenovus primary BC series that were enrolled to City Hospital in the period from 1988 to 1998. All patients' information was available including age ( $<70$ years, mean $=55$ years), menopausal status, tumour characteristics (grade, lymph node stage and size), vascular invasion and Nottingham prognostic index (NPI) [16]. The management protocol was uniform and included systemic hormonal therapy for those whose NPI was $>3.4$, and if they were premenopausal, Zoladex was added. The remaining ER-negative cases received chemotherapy in the form of cyclophosphamide, methotrexate and 5-fluorouracil.

The regular follow-up was collected and included BCspecific survival (time from the surgery until the patient die from or with BC with follow-up period of 15 years. Locoregional recurrence data were also available in this series. In addition, data on a large number of relevant biomarkers were also available as previously described [17-21].

\section{Immunohistochemistry (IHC)}

IHC staining for different MAPK markers was done using $4 \mu \mathrm{m}$ sections from TMA blocks as published before [22].

Details of primary antibodies and other relevant biomarkers included in this study are available in Online Resource. These biomarkers were used to molecularly characterise the series and further assess the biological function of MAPK activation in BC.

\section{Scoring of IHC}

TMA slides were manually scored using high-resolution digital images (NanoZoomer, Hamamatsu Photonics, Welwyn Garden City, UK) scanned at $\times 20$ magnification, by using a web-based interface (Distiller, Slidepath Ltd., Dublin, Ireland). H-score and percent were used to score the markers. The median scores and X-tile bioinformatics software (version 3.6.1, 2003-2005, Yale University, USA (http://x-tile.software.informer.com) were both used to derive optimal cut-off points for each marker [23].

\section{In vitro study}

The expression of MAPKs and the interaction with ER and HER 2 were evaluated in six different molecular classes of $\mathrm{BC}$ cell lines which included the following Wild-type MCF-7 (ER+/HER2-), MDA-MB-231 (ER-/HER2-), SKBR3 (ER-/HER2+) and BT474 (ER+/HER2+) and were obtained from the American Type Culture Collection (Manassas, VA, USA). In addition, MCF-7 (ER+) and MDA-231 (ER-) were stably transfected with HER2 gene to assess the impact of HER2 on MAPK activation in BC in relation to ER status [24]. The HER2 transfection has been confirmed by WB (Online Resource).

\section{Reverse phase protein microarray (RPPA)}

RPPA has been established before [25-28]. In this study, lysates from the six used BC cell lines were used to evaluate the expression of MAPK signalling intermediates. All primary antibodies were evaluated for specificity using 
Table 1 The associations between MAPKs and clinicopathological variables in breast cancer

\begin{tabular}{|c|c|c|c|c|c|c|c|c|c|}
\hline & \multicolumn{3}{|l|}{ Pan ERK1/2 } & \multicolumn{3}{|c|}{ Nuclear p-ERK1/2 } & \multicolumn{3}{|c|}{ Cytoplasmic-p-ERK1/2 } \\
\hline & Low $N(\%)$ & High $N(\%)$ & $p$ value & Low $N(\%)$ & $\operatorname{High} N(\%)$ & $p$ value & Low $N(\%)$ & High $N(\%)$ & $p$ value \\
\hline \multicolumn{10}{|l|}{ Grade } \\
\hline 1 & $85(14)$ & 99 (17) & $\mathbf{0 . 0 3 1}$ & $54(9)$ & $118(20)$ & $<0.0001$ & $52(10)$ & $119(20)$ & 0.001 \\
\hline 2 & $199(32)$ & $203(36)$ & & $156(28)$ & $241(41)$ & & $172(32)$ & $220(36)$ & \\
\hline 3 & $338(54)$ & $268(47)$ & & $352(63)$ & $235(39)$ & & $310(58)$ & $271(44)$ & \\
\hline \multicolumn{10}{|c|}{ Lymph node stage } \\
\hline 1 & $379(61)$ & $343(60)$ & 0.085 & $343(61)$ & $118(20)$ & NS & $313(58)$ & $384(63)$ & NS \\
\hline 2 & $180(29)$ & $188(33)$ & & $170(30)$ & $241(41)$ & & $164(31)$ & $178(29)$ & \\
\hline 3 & $63(10)$ & $40(7)$ & & $49(9)$ & $235(39)$ & & $57(11)$ & $48(8)$ & \\
\hline \multicolumn{10}{|l|}{ Tumour size } \\
\hline$<2 \mathrm{CM}$ & $290(47)$ & $301(53)$ & 0.029 & $242(43)$ & $324(54)$ & $<0.0001$ & $222(42)$ & $336(55)$ & $<0.0001$ \\
\hline$\geq 2 \mathrm{CM}$ & $334(53)$ & $269(47)$ & & $321(57)$ & $272(46)$ & & $312(58)$ & $277(45)$ & \\
\hline \multicolumn{10}{|c|}{ Lymphovascular invasion } \\
\hline No & $399(65)$ & $381(67)$ & & $357(64)$ & $399(67)$ & & $322(61)$ & $425(70)$ & 0.001 \\
\hline Definite & $219(35)$ & $190(33)$ & NS & $202(36)$ & $192(33)$ & NS & $210(39)$ & 181(30) & \\
\hline \multicolumn{10}{|l|}{ NPI } \\
\hline GPG & $170(28)$ & $183(34)$ & 0.015 & $121(23)$ & $209(37)$ & $<0.0001$ & $122(24)$ & $205(35)$ & $<0.0001$ \\
\hline MPG & $324(54)$ & $293(54)$ & & $314(59)$ & $293(51)$ & & $286(56)$ & $317(54)$ & \\
\hline \multirow[t]{3}{*}{ PPG } & $106(18)$ & $66(12)$ & & $101(19)$ & $70(12)$ & & $104(20)$ & $64(11)$ & \\
\hline & \multicolumn{3}{|l|}{ Pan JNK1/2 } & \multicolumn{3}{|l|}{$\mathrm{p}-\mathrm{JNK} 1 / 2$} & \multicolumn{3}{|l|}{ Pan p38 } \\
\hline & Low $N(\%)$ & $\operatorname{High} N(\%)$ & $p$ value & Low $N(\%)$ & High $N(\%)$ & $p$ value & Low $N(\%)$ & $\operatorname{High} N(\%)$ & $p$ value \\
\hline \multicolumn{10}{|l|}{ Grade } \\
\hline 1 & $100(17)$ & $104(19)$ & NS & $26(11)$ & $131(16)$ & $<0.0001$ & $26(11)$ & $131(16)$ & 0.002 \\
\hline 2 & $216(36)$ & $168(32)$ & & $45(20)$ & $308(37)$ & & $45(20)$ & 308 (37) & \\
\hline 3 & $277(47)$ & $260(49)$ & & $156(69)$ & $388(50)$ & & $156(69)$ & $388(50)$ & \\
\hline \multicolumn{10}{|c|}{ Lymph node stage } \\
\hline 1 & $398(65)$ & $356(65)$ & NS & $140(62)$ & $498(60)$ & NS & $140(62)$ & $498(60)$ & NS \\
\hline 2 & $169(8)$ & $150(27)$ & & $66(29)$ & $260(32)$ & & $66(29)$ & $260(32)$ & \\
\hline 3 & $46(7)$ & $42(8)$ & & $21(9)$ & $69(8)$ & & $21(9)$ & $69(8)$ & \\
\hline \multicolumn{10}{|c|}{ Tumour size } \\
\hline$<2 \mathrm{CM}$ & $283(50)$ & $274(55)$ & 0.079 & $93(40)$ & $427(52)$ & 0.003 & $93(40)$ & $427(52)$ & NS \\
\hline$>2 \mathrm{CM}$ & $286(50)$ & $223(45)$ & & $137(60)$ & $401(48)$ & & $137(60)$ & $401(48)$ & \\
\hline \multicolumn{10}{|c|}{ Lymphovascular invasion } \\
\hline No & $376(67)$ & $344(69)$ & NS & $153(67)$ & $540(66)$ & NS & $153(67)$ & $540(66)$ & NS \\
\hline Definite & $187(33)$ & $152(31)$ & & $76(33)$ & $282(34)$ & & $76(33)$ & $282(34)$ & \\
\hline \multicolumn{10}{|l|}{ NPI } \\
\hline GPG & $192(33)$ & $181(35)$ & NS & $47(21)$ & $255(32)$ & 0.002 & $47(21)$ & $255(32)$ & 0.013 \\
\hline MPG & $303(53)$ & $266(52)$ & & $132(60)$ & $435(55)$ & & $132(60)$ & $435(55)$ & \\
\hline \multirow[t]{3}{*}{ PPG } & 79 (14) & 69 (13) & & $42(19)$ & $103(13)$ & & $42(19)$ & $103(13)$ & \\
\hline & \multicolumn{3}{|l|}{ p-p38 } & \multicolumn{3}{|l|}{$\mathrm{p}-\mathrm{C}-\mathrm{JUN}$} & \multicolumn{3}{|l|}{ p-ATF2 } \\
\hline & Low $N(\%)$ & High $N(\%)$ & $p$ value & Low $N(\%)$ & High $N(\%)$ & $p$ value & Low $N(\%)$ & High $N(\%)$ & $p$ value \\
\hline Grade & & & & & & & & & \\
\hline 1 & $120(13)$ & $82(21)$ & $<0.0001$ & $62(14)$ & $135(16)$ & $<0.0001$ & $114(12)$ & $81(24)$ & $<0.0001$ \\
\hline 2 & $293(31)$ & $166(43)$ & & $119(26)$ & $313(38)$ & & $303(32)$ & $139(42)$ & \\
\hline 3 & $525(56)$ & $142(36)$ & & $268(60)$ & $380(46)$ & & $539(56)$ & $114(34)$ & \\
\hline Lymph nod & stage & & & & & & & & \\
\hline
\end{tabular}


Table 1 continued

\begin{tabular}{|c|c|c|c|c|c|c|c|c|c|}
\hline & \multicolumn{3}{|l|}{ p-p38 } & \multicolumn{3}{|l|}{ p-C-JUN } & \multicolumn{3}{|l|}{ p-ATF2 } \\
\hline & Low $N(\%)$ & High $N(\%)$ & $p$ value & Low $N(\%)$ & High $N(\%)$ & $p$ value & Low $N(\%)$ & High $N(\%)$ & $p$ value \\
\hline 1 & $550(59)$ & $261(67)$ & 0.008 & $266(59)$ & $517(62)$ & NS & $571(60)$ & $208(62)$ & NS \\
\hline 2 & $310(33)$ & $96(24)$ & & $143(32)$ & $251(30)$ & & $311(32)$ & $92(28)$ & \\
\hline 3 & $78(8)$ & $34(9)$ & & $40(9)$ & $61(8)$ & & $74(8)$ & $35(10)$ & \\
\hline \multicolumn{10}{|c|}{ Tumour size } \\
\hline$<2 \mathrm{CM}$ & $423(45)$ & $225(58)$ & $<0.0001$ & $206(46)$ & $421(51)$ & 0.080 & $451(47)$ & $173(52)$ & NS \\
\hline$>2 \mathrm{CM}$ & $517(55)$ & $166(42)$ & & $245(54)$ & $408(49)$ & & $507(53)$ & $162(48)$ & \\
\hline \multicolumn{10}{|c|}{ Lymphovascular invasion } \\
\hline No & $602(65)$ & $284(73)$ & 0.002 & $278(62)$ & $566(69)$ & 0.015 & $611(64)$ & $234(71)$ & $\mathbf{0 . 0 3 2}$ \\
\hline Definite & $332(35)$ & $104(27)$ & & $171(38)$ & $258(31)$ & & $344(36)$ & $98(29)$ & \\
\hline \multicolumn{10}{|l|}{ NPI } \\
\hline GPG & $235(26)$ & $159(43)$ & $<0.0001$ & $114(26)$ & $264(33)$ & 0.001 & $240(26)$ & $132(41)$ & $<0.0001$ \\
\hline MPG & $508(57)$ & 177 (47) & & $238(55)$ & $426(54)$ & & $530(58)$ & $143(45)$ & \\
\hline PPG & $156(17)$ & 38 (10) & & 84 (19) & 100 (13) & & 149 (16) & 44 (14) & \\
\hline
\end{tabular}

$p$ values in bold denote significant ones $(<0.050)$, borderline: $(0.05-0.09)$, NS: $>0.09)$ and the same in all tables

$N P I$ Nottingham prognostic index, $G P G$ good prognostic group, $M P G$ moderate prognostic group, $P P G$ poor prognostic group

Western blotting before using in RPPA as described before $[22,25]$.

\section{Statistical analysis}

The statistical analysis was performed using Statistical Package for Social Sciences SPSS v21. Chi-square test was used to test the associations between categorical data. Kaplan-Meier test was used for univariate analysis. Kruskal-Wallis test was used to test the associations for the results of RPPA. Moreover, Spearman's rank correlations were used. A two-tailed $p$ value of less than 0.05 was considered significant for all the used statistical tests.

\section{Results}

\section{MAPK expression in BC tissue}

In this study, 16 MAPKs members were investigated. Specificity of MAPKs proteins was confirmed by Western blot which revealed specific band for each protein (Online Resource). IHC staining of MAPKs (pan and phosphorylated (p) ERK1/2, pan JNK1/2, p-JNK1/2, pan p38, p-p38, p-ATF2 and p-C-JUN) revealed nuclear expression of phosphorylated proteins except p-ERK1/2 which showed both nuclear and cytoplasmic expression. The total/unphosphorylated forms showed cytoplasmic expression. All
MAPKs proteins showed an equivocal expression in normal breast tissue, DCIS and BC tissue included within the TMA cores at varying degrees ranging from negative to strong positivity (Online Resource).

Cut-off of positivity was chosen for each marker to assess its association with other variables. There were positive correlations between different members of MAPKs using continuous data as well as dichotomised variables (Online Resource).

\section{The association between MAPKs and clinicopathological variables}

Expression of MAPK proteins showed positive correlations with clinicopathological features characteristic of good prognosis including lower grade, early stage, smaller tumour size, absent lymphovascular invasion and lower NPI scores (Table 1).

\section{The association between MAPKs and key BC biomarkers}

There was significant correlation with ER and HER2 in addition to other key $\mathrm{BC}$ biomarkers including the proliferation marker KI67-LI and the apoptosis markers BCL2 and p53 (Table 2). Pan ERK1/2 showed strong positive association with ER and BCL2 but only showed borderline negative association with KI67-LI and p53. p-ERK1/2 was 
Table 2 Associations between MAPKs and biological markers in the whole series

\begin{tabular}{|c|c|c|c|c|c|c|c|c|c|}
\hline & \multicolumn{3}{|l|}{ Pan ERK1/2 } & \multicolumn{3}{|c|}{ Nuclear p-ERK1/2 } & \multicolumn{3}{|c|}{ Cytoplasmic-p-ERK1/2 } \\
\hline & Low $N(\%)$ & $\operatorname{High} N(\%)$ & $p$ value & Low $N(\%)$ & $\operatorname{High} N(\%)$ & $p$ value & Low $N(\%)$ & High $N(\%)$ & $p$ value \\
\hline \multicolumn{10}{|l|}{ ER } \\
\hline Negative & $176(28)$ & $112(20)$ & $<0.0001$ & $186(33)$ & $102(17)$ & $<0.0001$ & $146(28)$ & $139(23)$ & 0.066 \\
\hline Positive & $444(72)$ & $460(80)$ & & $373(67)$ & $487(83)$ & & $385(72)$ & $468(77)$ & \\
\hline \multicolumn{10}{|l|}{ HER2 } \\
\hline Negative & $516(87)$ & $480(87)$ & NS & $443(83)$ & $516(90)$ & $<0.0001$ & $442(86)$ & $506(86)$ & NS \\
\hline Positive & $75(13)$ & $70(13)$ & & 93 (17) & $58(10)$ & & $71(14)$ & 79 (14) & \\
\hline \multicolumn{10}{|l|}{ KI67-LI } \\
\hline Low & $200(39)$ & $208(46)$ & 0.050 & $153(34)$ & $224(47)$ & $<0.0001$ & 159 (37) & $233(47)$ & 0.003 \\
\hline High & $307(61)$ & $247(54)$ & & $304(66)$ & $250(53)$ & & $266(63)$ & $263(53)$ & \\
\hline \multicolumn{10}{|l|}{ P53 } \\
\hline Negative & $400(68)$ & $405(73)$ & 0.064 & $361(68)$ & $416(74)$ & 0.032 & $351(69)$ & $416(72)$ & NS \\
\hline Positive & $185(32)$ & 147 (27) & & $172(32)$ & 149 (26) & & $160(31)$ & $158(28)$ & \\
\hline \multicolumn{10}{|l|}{ BCL2 } \\
\hline Negative & 199 (47) & $134(32)$ & $<0.0001$ & $113(37)$ & $77(24)$ & $<0.0001$ & $174(46)$ & $183(41)$ & NS \\
\hline \multirow[t]{3}{*}{ Positive } & $225(53)$ & $283(68)$ & & $196(63)$ & $239(76)$ & & $208(54)$ & $269(59)$ & \\
\hline & \multicolumn{3}{|l|}{ Pan JNK1/2 } & \multicolumn{3}{|l|}{ p-JNK1/2 } & \multicolumn{3}{|l|}{ Pan p38 } \\
\hline & Low $N(\%)$ & High $N(\%)$ & $\overline{p \text { value }}$ & Low $N(\%)$ & High $N(\%)$ & $\overline{p \text { value }}$ & Low $N(\%)$ & $\operatorname{High} N(\%)$ & $\overline{p \text { value }}$ \\
\hline \multicolumn{10}{|l|}{ ER } \\
\hline Negative & $130(22)$ & 149 (28) & 0.015 & $94(41)$ & $189(23)$ & $<0.0001$ & $221(31)$ & 89 (19) & $<0.0001$ \\
\hline Positive & $466(78)$ & $382(72)$ & & $136(59)$ & $633(77)$ & & $492(69)$ & $387(81)$ & \\
\hline \multicolumn{10}{|l|}{ HER2 } \\
\hline Negative & $478(88)$ & $406(85)$ & 0.092 & $184(83)$ & $690(87)$ & NS & $594(88)$ & $385(87)$ & NS \\
\hline Positive & $63(12)$ & $73(15)$ & & $37(17)$ & $103(13)$ & & $81(12)$ & $59(13)$ & \\
\hline \multicolumn{10}{|l|}{ KI67-LI } \\
\hline Low & 199 (44) & $172(45)$ & NS & $58(31)$ & $292(44)$ & 0.001 & $594(88)$ & $385(87)$ & 0.058 \\
\hline High & $255(56)$ & $213(55)$ & & $127(69)$ & $368(56)$ & & $81(12)$ & $59(13)$ & \\
\hline \multicolumn{10}{|l|}{ P53 } \\
\hline Negative & 394 (74) & 331 (69) & NS & $149(68)$ & $563(72)$ & NS & $460(69)$ & $331(74)$ & 0.060 \\
\hline Positive & $141(26)$ & $149(31)$ & & $70(32)$ & $222(28)$ & & $205(31)$ & $113(26)$ & \\
\hline \multicolumn{10}{|l|}{ BCL2 } \\
\hline Negative & $150(38)$ & $183(48)$ & 0.004 & $90(52)$ & $247(41)$ & 0.005 & $224(46)$ & $114(34)$ & 0.001 \\
\hline \multirow[t]{3}{*}{ Positive } & $246(62)$ & $197(52)$ & & $82(48)$ & $368(59)$ & & $259(54)$ & $221(66)$ & \\
\hline & \multicolumn{3}{|l|}{ p-p38 } & \multicolumn{3}{|l|}{ p-C-JUN } & \multicolumn{3}{|l|}{ p-ATF2 } \\
\hline & Low $N(\%)$ & High $N(\%)$ & $p$ value & Low $N(\%)$ & High $N(\%)$ & $p$ value & Low $N(\%)$ & $\operatorname{High} N(\%)$ & $p$ value \\
\hline ER & & & & & & & & & \\
\hline Negative & $246(26)$ & $70(18)$ & 0.002 & $130(29)$ & $178(22)$ & 0.005 & $260(27)$ & $51(15)$ & 0.003 \\
\hline Positive & $692(74)$ & $317(82)$ & & $321(71)$ & 645 (78) & & $693(73)$ & $281(85)$ & \\
\hline HER 2 & & & & & & & & & \\
\hline Negative & 777 (86) & 329 (89) & NS & $371(86.3)$ & $687(86.5)$ & NS & 777 (85) & $299(92)$ & 0.001 \\
\hline Positive & $128(14)$ & $42(11)$ & & $59(13.7)$ & 107 (13.5) & & $138(15)$ & $25(8)$ & \\
\hline KI67-LI & & & & & & & & & \\
\hline Low & $292(39)$ & $161(53)$ & $<0.0001$ & 127 (35.6) & $304(45.8)$ & 0.002 & $289(38)$ & $144(53)$ & $<0.0001$ \\
\hline High & $463(61)$ & $142(47)$ & & $230(64.4)$ & $360(54.2)$ & & $466(62)$ & $130(47)$ & \\
\hline P53 & & & & & & & & & \\
\hline Negative & $637(70)$ & 267 (73) & NS & $302(70.2)$ & $564(71.5)$ & NS & $634(70)$ & $243(76)$ & 0.025 \\
\hline
\end{tabular}


Table 2 continued

\begin{tabular}{|c|c|c|c|c|c|c|c|c|c|}
\hline & \multicolumn{3}{|l|}{ p-p38 } & \multicolumn{3}{|l|}{ p-C-JUN } & \multicolumn{3}{|l|}{ p-ATF2 } \\
\hline & Low $N(\%)$ & High $N(\%)$ & $p$ value & Low $N(\%)$ & High $N(\%)$ & $p$ value & Low $N(\%)$ & High $N(\%)$ & $p$ value \\
\hline Positive & $269(30)$ & $97(27)$ & & $128(29.8)$ & $225(28.5)$ & & $277(30)$ & $76(24)$ & \\
\hline \multicolumn{10}{|l|}{ BCL2 } \\
\hline Negative & $297(43)$ & $99(36)$ & 0.037 & $138(43.5)$ & 249 (41.2) & NS & 305 (44) & $84(34)$ & 0.009 \\
\hline Positive & 391 (57) & 177 (64) & & $179(56.5)$ & $355(58.8)$ & & $396(56)$ & $163(66)$ & \\
\hline
\end{tabular}

positively associated with ER and negatively with BCL2 but only its nuclear form showed a positive association with BCL2 and a negative association with HER2 and p53. Pan JNK1/2 was associated with downregulation of ER and BCL2; however, its phosphorylated form was associated with increased expression of ER, BCL2 and with downregulation of KI67-LI. p-p38 and its total form were positively associated with ER and BCL2 and negatively with KI67-LI.

p-ATF2 and p-C-JUN, which are downstream markers of the MAPK pathway, showed positive associations with ER and negative association with KI67-LI. p-ATF2 also showed positive association with BCL2 and negative with HER2 and $\mathrm{p} 53$.

Within ER+ tumours, most of the associations observed in the whole series remained significant including nuclear p-ERK1/2, p-p38 and p-ATF2 (Table 3). When the ER+ group was further stratified based on HER2 expression, some associations were maintained in the ER+/HER2subgroup (Online Resource) but not in the ER+/HER2+ tumours. Interestingly, when the analysis was restricted to HER2 - tumours, the associations observed in the whole cohort and in the ER+ class were maintained (Online Resource). Importantly, when the analysis was restricted to ER - class, pan-ERK1/2 and p-p38 were associated positively with HER2 ( $p=0.006,0.003$ respectively; Online Resource).

\section{Outcome analysis}

Univariate survival analyses revealed that MAPKs (pan ERK1/2, nuclear p-ERK1/2, p-JNK1/2, p-p38, p-C-JUN and p-ATF2) were associated with better outcome in terms of prolonged BCSS (Fig. 1). ERK1/2 and N-p-ERK1/2 showed better associations with prolong survival within ER+BC (Fig. 2).

\section{Proteomic analysis of $\mathrm{BC}$ cell lines}

RPPA was consistent with IHC results and confirmed that MAPK expression was higher in ER+ compared to ERcell lines and in the ER+/HER2- cell lines compared to
ER+/HER2 +. In the ER - cell lines, there was a trend towards positive correlation between MAPs and HER2 expression. Interestingly, most of these MAPKs showed higher expression in ER+HER2- compared to ER-HER2- cell line.

For example, there was a significant increase in the expression of p-C-RAF in ER+HER2- (MCF-7) cell line compared to ER+HER2+ transfected (T) while in the ER - cell lines there was high expression of this protein in ER-HER2+ cell line compared to ER-HER2 - and the difference between these two cell lines was statistically significant. Regarding MEK1/2, which is the downstream mediator of p-C-RAF, the same association was noticed. Similarly, ERK1/2 which is the downstream mediator of MEK1/2, showed an increase in its expression in ER+HER2 - compared to ER+HER2+ and high expression in ER-HER2+ (W and T) compared to ER-HER2cell lines (Fig. 3). The details for the expression of all the MAPK markers are shown in (Online Resource), with all the $p$ values for the comparison of the expression levels between the different cell lines.

\section{Discussion}

Several studies have emphasised the role of MAPKs in cancer progression [13, 29-31]. The functions of MAPKs in $\mathrm{BC}$ appear to be complex owing to several cellular responses that they modulate and their interaction with different pathways including the key BC genes ER and HER2. In the current study, the role of MAPKs in BC and how the expression of ER and HER2 might influence their function were investigated using a large panel of MAPK proteins and the results were validated in vitro using RPPA and different BC cell lines. The results showed that generally most of MAPKs are associated with good prognostic features in the whole series and in the ER+ tumours. MAPKs are mainly related to ER expression and this finding was observed using IHC and validated by RPPA.

Our results are consistent with others [32] who demonstrated that ERK1/2 and p-ERK1/2 were associated 
Table 3 Associations between MAPK and biological markers in ER-positive tumours

\begin{tabular}{|c|c|c|c|c|c|c|c|c|c|}
\hline & \multicolumn{3}{|l|}{ Pan ERK1/2 } & \multicolumn{3}{|l|}{ N-p-ERK1/2 } & \multicolumn{3}{|l|}{ C-p-ERK1/2 } \\
\hline & Low $N(\%)$ & $\operatorname{High} N(\%)$ & $p$ value & Low $N(\%)$ & High $N(\%)$ & $p$ value & Low $N(\%)$ & High $N(\%)$ & $p$ value \\
\hline \multicolumn{10}{|l|}{ HER 2} \\
\hline Negative & 379 (89) & 407 (92) & NS & $315(87)$ & $440(93)$ & 0.005 & 337 (90) & 409 (91) & NS \\
\hline Positive & $46(11)$ & $37(8)$ & & 47 (13) & $34(7)$ & & $38(10)$ & $42(9)$ & \\
\hline \multicolumn{10}{|l|}{ KI67-LI } \\
\hline Low & $179(50)$ & $190(52)$ & NS & $137(45)$ & $216(55)$ & 0.005 & $141(46)$ & $211(55)$ & 0.010 \\
\hline High & $180(50)$ & $174(4.8)$ & & $171(55)$ & $175(45)$ & & $169(54)$ & $170(45)$ & \\
\hline \multicolumn{10}{|l|}{ P53 } \\
\hline Negative & $325(77)$ & $364(82)$ & 0.093 & $282(79)$ & $368(79)$ & NS & $291(78)$ & $351(79)$ & NS \\
\hline Positive & $96(23)$ & $81(18)$ & & $76(21)$ & $99(21)$ & & $81(22)$ & $92(21)$ & \\
\hline \multicolumn{10}{|l|}{ BCL2 } \\
\hline Negative & $101(33)$ & $81(23)$ & 0.005 & $105(36)$ & $87(25)$ & 0.001 & $95(33)$ & $95(27)$ & NS \\
\hline \multirow[t]{3}{*}{ Positive } & $206(67)$ & $269(77)$ & & $186(64)$ & $267(75)$ & & $191(67)$ & $254(73)$ & \\
\hline & \multicolumn{3}{|l|}{ Pan JNK1/2 } & \multicolumn{3}{|l|}{ p-JNK1/2 } & \multicolumn{3}{|l|}{ Pan p38 } \\
\hline & Low $N(\%)$ & $\operatorname{High} N(\%)$ & $p$ value & Low $N(\%)$ & High $N(\%)$ & $p$ value & Low $N(\%)$ & $\operatorname{High} N(\%)$ & $p$ value \\
\hline \multicolumn{10}{|l|}{ HER2 } \\
\hline Negative & $386(91)$ & $313(90)$ & NS & $115(87)$ & $553(91)$ & NS & $434(92)$ & $328(90)$ & NS \\
\hline Positive & $36(9)$ & $33(10)$ & & $17(13)$ & $57(9)$ & & $35(8)$ & $36(10)$ & \\
\hline \multicolumn{10}{|l|}{ KI67-LI } \\
\hline Low & $185(53)$ & $152(54)$ & NS & $48(44)$ & $265(53)$ & 0.098 & $208(53)$ & $158(52)$ & NS \\
\hline High & $164(47)$ & $127(46)$ & & $61(56)$ & $237(47)$ & & $187(47)$ & $144(48)$ & \\
\hline \multicolumn{10}{|l|}{ P53 } \\
\hline Negative & $338(80)$ & $267(7.8)$ & NS & $108(83)$ & $475(78)$ & NS & $373(81)$ & $295(81)$ & NS \\
\hline Positive & $83(20)$ & $76(22)$ & & $22(17)$ & $131(22)$ & & $88(19)$ & 69 (19) & \\
\hline \multicolumn{10}{|l|}{ BCL2 } \\
\hline Negative & $85(27)$ & $84(31)$ & NS & $36(33)$ & $131(28)$ & NS & $105(31)$ & $66(24)$ & 0.057 \\
\hline \multirow[t]{3}{*}{ Positive } & $231(73)$ & $186(69)$ & & $73(67)$ & $342(72)$ & & $237(69)$ & $211(76)$ & \\
\hline & \multicolumn{3}{|l|}{ p-p38 } & \multicolumn{3}{|l|}{ p-C-JUN } & \multicolumn{3}{|l|}{ p-ATF2 } \\
\hline & Low $N(\%)$ & $\operatorname{High} N(\%)$ & $p$ value & Low $N(\%)$ & High $N(\%)$ & $p$ value & Low $N(\%)$ & $\operatorname{High} N(\%)$ & $p$ value \\
\hline \multicolumn{10}{|l|}{ HER2 } \\
\hline Negative & $593(88)$ & $283(95)$ & 0.001 & $273(89)$ & $563(90)$ & NS & $594(89)$ & $256(94)$ & 0.010 \\
\hline Positive & $78(12)$ & $15(5)$ & & $32(11)$ & $59(10)$ & & $73(11)$ & $15(6)$ & \\
\hline \multicolumn{10}{|l|}{ KI67-LI } \\
\hline Low & $264(47)$ & $147(60)$ & 0.001 & $113(44)$ & $277(54)$ & 0.011 & $256(47)$ & $136(58)$ & 0.003 \\
\hline High & $295(53)$ & $97(40)$ & & $143(56)$ & $237(46)$ & & $292(53)$ & 97 (42) & \\
\hline \multicolumn{10}{|l|}{ P53 } \\
\hline Negative & $533(80)$ & $236(79)$ & NS & $248(81)$ & $488(79)$ & NS & $523(79)$ & $523(79)$ & NS \\
\hline Positive & $135(20)$ & $61(21)$ & & $58(19)$ & $128(21)$ & & $140(21)$ & $140(21)$ & \\
\hline \multicolumn{10}{|l|}{ BCL2 } \\
\hline Negative & $162(31)$ & $50(23)$ & 0.026 & $71(30)$ & $136(29)$ & NS & $153(29)$ & $55(27)$ & NS \\
\hline Positive & $360(69)$ & 168 (77) & & $163(70)$ & $333(71)$ & & $368(71)$ & $151(73)$ & \\
\hline
\end{tabular}

with good clinicopathological variables and that by $\mathrm{Hsu}$ et al. [33] who found that p-ERK1/2 is required for inducing apoptosis especially in MCF-7 and MDA-231 cell lines. The association between MAPKs and good prognostic variable may be related to their roles in inducing apoptosis. p-JNK1/2 also has been shown to be stimulated by stress or growth factors and either one can enhance p-JNK1/2 to stimulate apoptosis [34] and even p-JNK1/2 

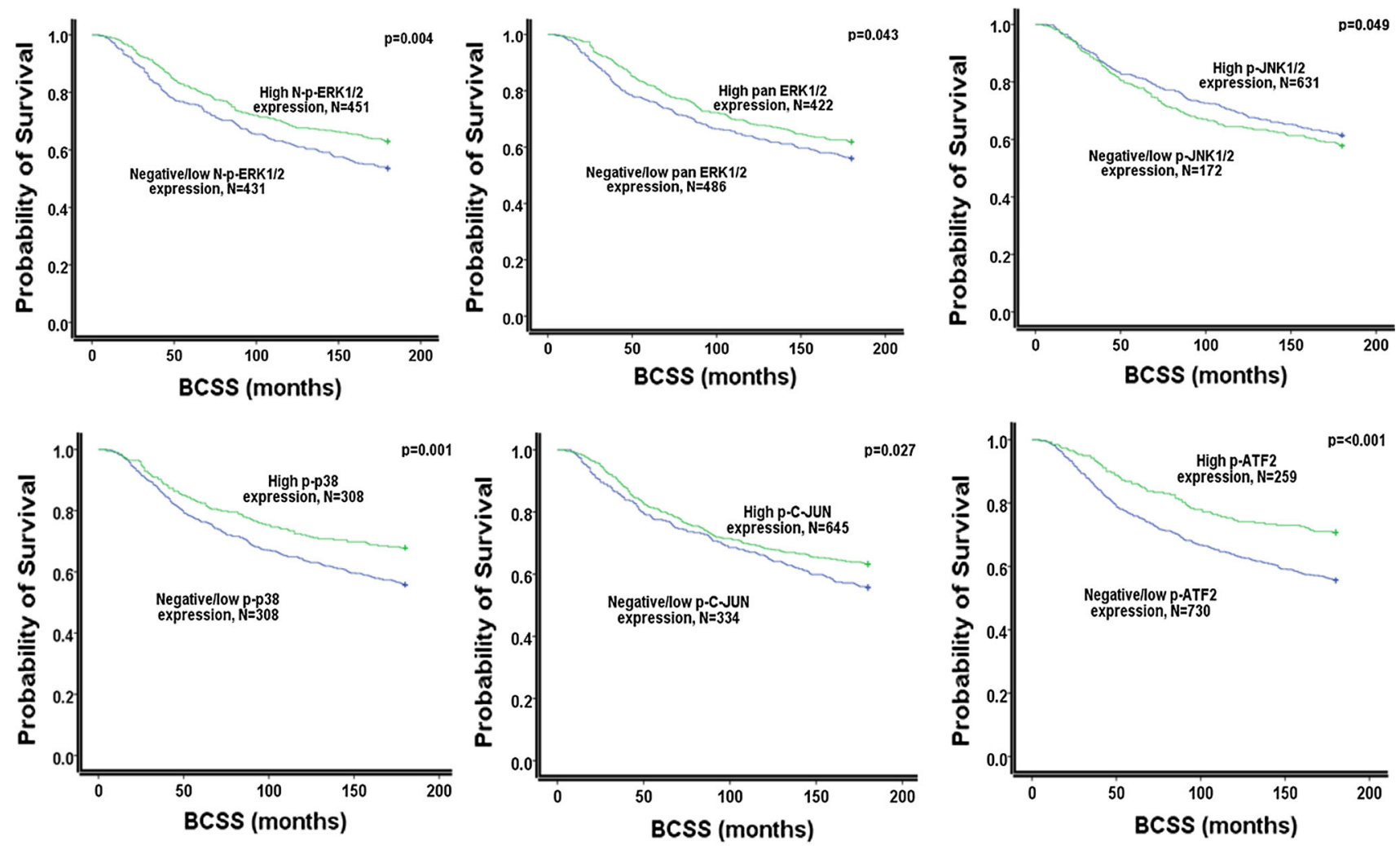

Fig. 1 This Kaplan-Meier survival curve illustrates that MAPK members including the nuclear p-ERK1/2, its total form ERK1/2, p-JNK1/2, p-p38 and the two transcription factors p-C-JUN and p-ATF2 are all associated significantly with prolonged BCSS in the whole series

augments cell death signalling in slowly growing MCF-7 cells under the influence of high estradiol level [35]. Interestingly, a drug "pseudolaric acid B" whose function mimics the upstream mediators of MAPKs was used in an in vitro experiment and this revealed the apoptotic function of JNK and its phosphorylated form upon activation [36]. Moreover, several studies have demonstrated the role of p-p38/MAPK in inducing apoptosis in BC and some suggested that this effect is mediated by TGF- $\beta$ [37-40]. p-ATF2 is thought to have dual functions independent of each other; the first is a tumour suppressor protein and the other function is related to DNA damage response pathway [41] and importantly, the former function has been confirmed by different studies [38, 42, 43]. The above results regarding the association between MAPKs and apoptosis were supported by our findings that MAPKs were positively associated with ER, BCL2 and negatively with HER2 (or no association with some of them), KI67-LI and p53.

Consistent with previous studies that assessed the prognostic value of individual MAPK members $[32,43,44]$, in this study we identified an association between MAPKs proteins and better outcome in terms of longer survival time. Importantly, in the current research, pan ERK1/2 and p-ERK1/2 showed an association with better outcome in patients with ER+ tumours who are candidate for endocrine therapy. Consistent with this, Busch et al. [45] have demonstrated that high p-ERK1/2 was an independent predictor of better outcome in tamoxifen-treated patients. Thus, this study implies that MAPKs could be prospective surrogate biomarkers in assessing benefit from endocrine therapy.

In the current study, RPPA results were consistent with IHC findings for those proteins used in both techniques and the findings were also consistent for those MAPK members that were used only in RPPA. In the ER-/HER2+ cell line and in line with our results, a previous study demonstrated that activation of MAPK is associated with loss of ER phenotype especially in those overexpressing HER2 [46]. Ostrakhovitch et al. [47] have found that in MDA-231 cell line where p53 is mutated or suppressed, the phosphorylation of ERK1/2 was strong. Meanwhile, p-p38 overexpression in ER- tumours is thought to be associated with proliferation and progression of cancer. This is attributed to the fact that p-p38 can mediate proliferation only in BC cells that express mutant p53 and not the wild one, a finding commonly seen in ER - tumours rather than ER+ tumours [48]. Interestingly, Creighton et al. [49] indicated that hyperactivation of MAPK leads to the loss of ER expression and plays a role in the generation of the ER- 
Fig. 2 Kaplan-Meier survival curve showing the associations of MAPKs (nuclear (N)p ERK1/ 2 and pan ERK1/2 are associated significantly with prolonged BCSS in ER-positive series
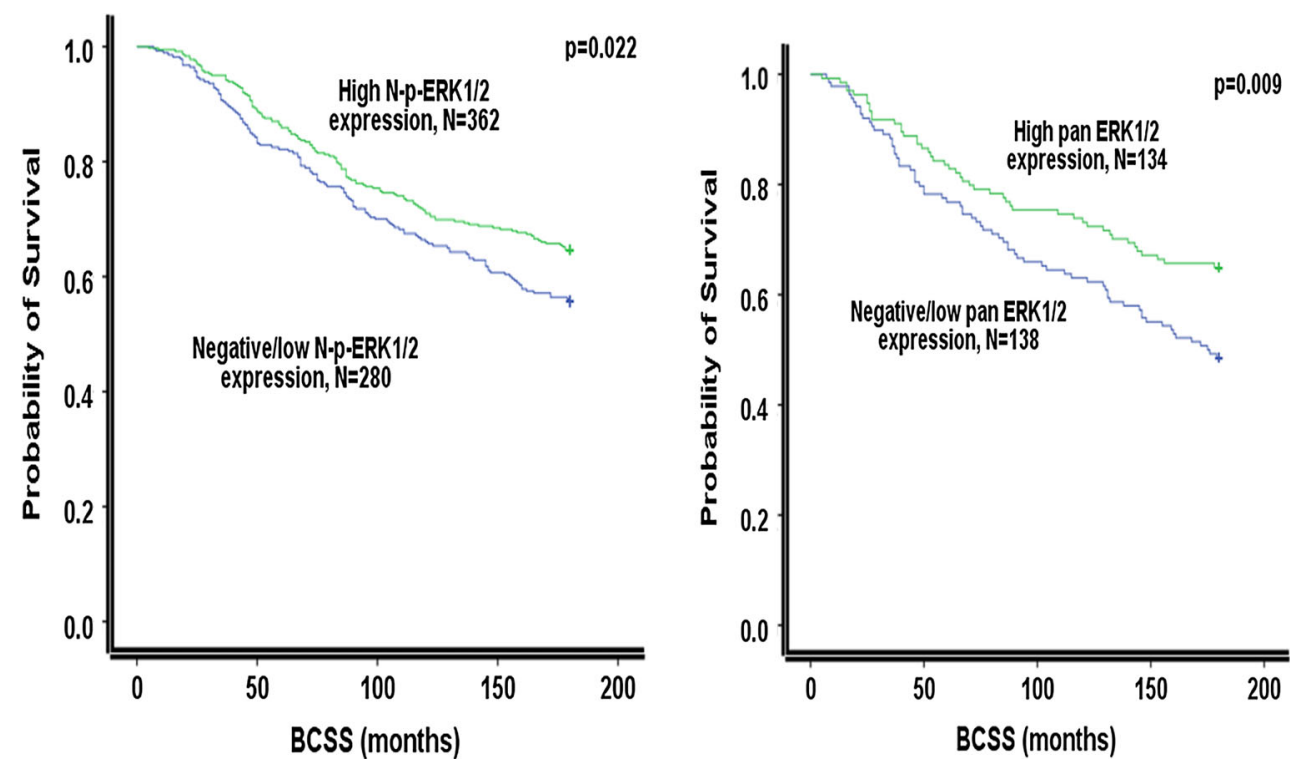

I)

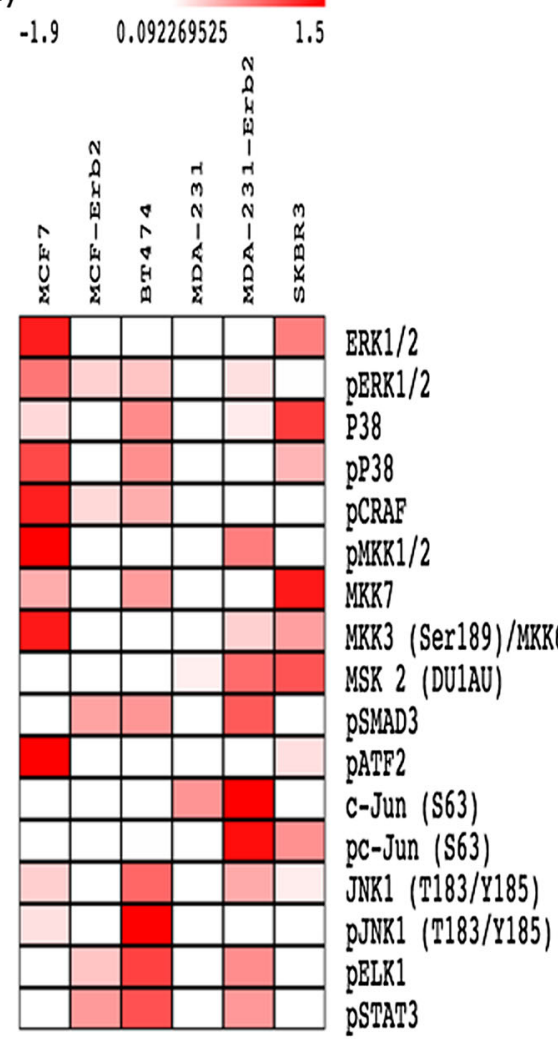

II)
A



C

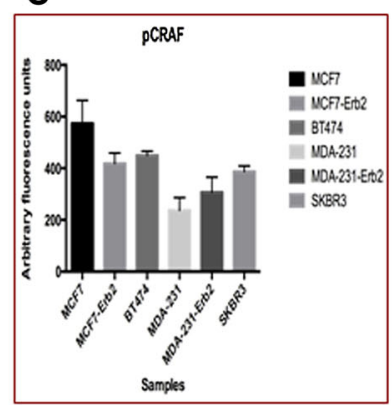

B

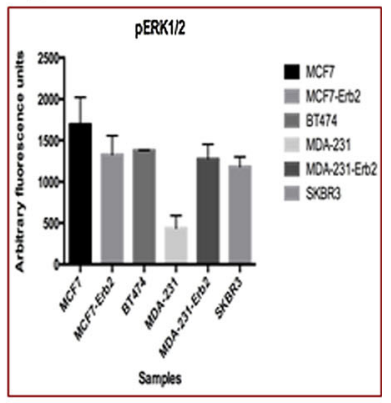

D

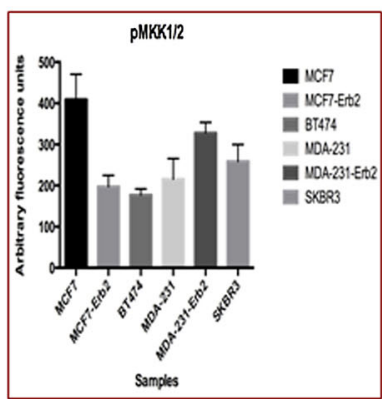

Fig. 3 Heat-map showing different MAPK pathway intermediates studied in six different breast cancer cell lines. Rows represent the different signalling molecules studied. White and red denote markers that are present at lower and higher levels, respectively. II: Bar graphs representing measurement of selected target proteins from the heat-map data, including a ERK1/2, b pERK1/2, c pCRAF and d pMKK1/2. Data are shown as individual sample data (mean of four technical replicates per sample) of three different experiments. Signal represents arbitrary fluorescence units; AFU, after background subtraction and normalisation to $\beta$-actin. Significance values were derived using the Kruskal-Wallis test 
negative phenotype. Although our study supports the differential expression of MAPKs between ER+ and ERphenotypes, it indicates that MAPKs play an important role in ER+ tumours and that their expression in ER+ tumours is associated with better outcome. Supporting this, Atanaskova et al. [50] have indicated that activation of ER by MAPK enhances the expression of ER-regulated genes, accelerates tumour growth, and that MAPK/ER crosstalk enhances ER-mediated signalling without diminishing sensitivity to the inhibitory effects of anti-estrogens. However, in the ER-negative subgroup, it has been reported that the oncogenic effect of MAPKs is related to HER2 overexpression [49], which further support our findings.

The role of MAPKs in enhancing cell cycle arrest is also encountered. This has been attributed to the fact that innate tumour suppressor mechanisms are activated in response to aberrant oncogenic stimulation and remarkably induce growth inhibition that is referred to as oncogenic-induced senescence [51,52]. In addition, accumulating evidence has demonstrated that sequestration of cytoplasmic ERK in the cytoplasm induced by proapoptotic molecules such as death-associated protein kinase could ultimately augment the apoptotic action of these proapoptotic proteins [53]. In this regard, a study revealed that feedback inhibitory signals are common from mutated RAS and RAF to the upstream mediators, which will omit further stimulation through this pathway being thoughtfully mediated by HDM2 and FOXO3 [54]. The previous findings might explain why high expression of MAPKs in ER+ tumours was associated with good features and outcome in our IHC findings and why the function is different in ER + and ER - cell lines by using RPPA. In addition, another reason which can explain the dual behaviour of these MAPKs is that they function in a cell context-specific and cell typespecific way to mediate signals that can lead to diverse cellular functions. Furthermore, the function of these MAPKs is influenced by their crosstalk and interaction with other pathways which can influence their behaviour [9-11].

Despite the limitations of this study and type of techniques employed, the findings of this study collectively together with other previous publications mentioned above support the tumour suppressor role of MAPK kinase pathway in $\mathrm{BC}$ and that active pathway is associated with variables of good prognosis and better outcome. In addition, our results provide further evidence that ER is the main player related to the function of MAPK in BC; however, when HER2 is overexpressed, the role of ER becomes less significant and there is some evidence that HER2 can play a major role in controlling the MAPK pathway activation in ER-negative tumours.

In conclusion, our study illustrated the role of MAPKs and their signalling in BC and demonstrated that MAPKs are associated with good prognostic features and show differential expression within ER+ and ER- groups.
Acknowledgments Dr. Dena A J Ahmad is funded by the higher committee of educational development in Iraq.

\section{Compliance with ethical standards}

Conflict of interest The authors declare that they have no conflict of interest.

Ethical standards This study was approved by the Nottingham Research Ethics Committee.

Open Access This article is distributed under the terms of the Creative Commons Attribution 4.0 International License (http://crea tivecommons.org/licenses/by/4.0/), which permits unrestricted use, distribution, and reproduction in any medium, provided you give appropriate credit to the original author(s) and the source, provide a link to the Creative Commons license, and indicate if changes were made.

\section{References}

1. Dhillon AS et al (2007) MAP kinase signalling pathways in cancer. Oncogene 26(22):3279-3290

2. McCubrey JA et al (2007) Roles of the Raf/MEK/ERK pathway in cell growth, malignant transformation and drug resistance. Biochim Biophys Acta 1773(8):1263-1284

3. Chappell WH et al (2011) Ras/Raf/MEK/ERK and PI3K/PTEN/ Akt/mTOR inhibitors: rationale and importance to inhibiting these pathways in human health. Oncotarget 2(3):135-164

4. Neel NF et al (2011) The RalGEF-Ral effector signaling network: the road less traveled for anti-ras drug discovery. Genes Cancer 2(3):275-287

5. Rajalingam K et al (2007) Ras oncogenes and their downstream targets. Biochim Biophys Acta 1773(8):1177-1195

6. Ramos JW (2008) The regulation of extracellular signal-regulated kinase (ERK) in mammalian cells. Int $\mathrm{J}$ Biochem Cell Biol 40(12):2707-2719

7. Johannessen CM et al (2010) COT drives resistance to RAF inhibition through MAP kinase pathway reactivation. Nature 468(7326):968-972

8. Schaeffer HJ, Weber MJ (1999) Mitogen-activated protein kinases: specific messages from ubiquitous messengers. Mol Cell Biol 19(4):2435-2444

9. Kyriakis JM, Avruch J (2001) Mammalian mitogen-activated protein kinase signal transduction pathways activated by stress and inflammation. Physiol Rev 81(2):807-869

10. Karin M, Gallagher E (2005) From JNK to pay dirt: jun kinases, their biochemistry, physiology and clinical importance. IUBMB Life 57(4-5):283-295

11. Weston CR, Davis RJ (2007) The JNK signal transduction pathway. Curr Opin Cell Biol 19(2):142-149

12. Merlin JL et al (2013) Expression and activation of P38 MAP kinase in invasive ductal breast cancers: correlation with expression of the estrogen receptor, HER2 and downstream signaling phosphorylated proteins. Oncol Rep 30(4):1943-1948

13. Huang L et al (2013) Prognostic and predictive value of Phosphop44/42 and pAKT in HER2-positive locally advanced breast cancer patients treated with anthracycline-based neoadjuvant chemotherapy. World J Surg Oncol 11:307

14. Kuo HT et al (2013) High nuclear phosphorylated extracellular signal-regulated kinase expression associated with poor differentiation, larger tumor size, and an advanced stage of breast cancer. Pol J Pathol 64(3):163-169 
15. McShane LM et al (2006) Reporting recommendations for tumor MARKer prognostic studies (REMARK). Breast Cancer Res Treat 100(2):229-235

16. Rakha EA et al (2008) Invasive lobular carcinoma of the breast: response to hormonal therapy and outcomes. Eur J Cancer 44(1):73-83

17. AbdEl-Rehim DM et al (2005) High-throughput protein expression analysis using tissue microarray technology of a large wellcharacterised series identifies biologically distinct classes of breast cancer confirming recent cDNA expression analyses. Int $\mathbf{J}$ Cancer 116(3):340-350

18. AbdEl-Rehim DM et al (2004) Expression and co-expression of the members of the epidermal growth factor receptor (EGFR) family in invasive breast carcinoma. Br J Cancer 91(8):1532-1542

19. Rakha EA et al (2006) Basal phenotype identifies a poor prognostic subgroup of breast cancer of clinical importance. Eur J Cancer 42(18):3149-3156

20. Rakha EA et al (2006) Prognostic markers in triple-negative breast cancer. Cancer 109(1):25-32

21. Rakha EA et al (2007) Prognostic markers in triple-negative breast cancer. Cancer 109(1):25-32

22. Jerjees DA et al (2015) The mammalian target of rapamycin complex 1 (mTORC1) in breast cancer: the impact of oestrogen receptor and HER2 pathways. Breast Cancer Res Treat 150(1):91-103

23. Camp RL, Dolled-Filhart M, Rimm DL (2004) X-tile: a new bioinformatics tool for biomarker assessment and outcome-based cut-point optimization. Clin Cancer Res 10(21):7252-7259

24. Dimri M et al (2007) Modeling breast cancer-associated c-Src and EGFR overexpression in human MECs: c-Src and EGFR cooperatively promote aberrant three-dimensional acinar structure and invasive behavior. Cancer Res 67(9):4164-4172

25. Negm OH et al (2014) A pro-inflammatory signalome is constitutively activated by $\mathrm{C} 33 \mathrm{Y}$ mutant TNF receptor 1 in TNF receptor-associated periodic syndrome (TRAPS). Eur J Immunol 44(7):2096-2110

26. Alshareeda AT et al (2014) SUMOylation proteins in breast cancer. Breast Cancer Res Treat 144(3):519-530

27. Aleskandarany MA et al (2014) Epithelial mesenchymal transition in early invasive breast cancer: an immunohistochemical and reverse phase protein array study. Breast Cancer Res Treat 145(2):339-348

28. Mannsperger HA et al (2010) RPPanalyzer: analysis of reversephase protein array data. Bioinformatics 26(17):2202-2203

29. Reddy KB et al (1999) Mitogen-activated protein kinase (MAPK) regulates the expression of progelatinase B (MMP-9) in breast epithelial cells. Int J Cancer 82(2):268-273

30. Liu QH et al (2015) Role of the ERK1/2 pathway in tumor chemoresistance and tumor therapy. Bioorg Med Chem Lett 25(2):192-197

31. Neuzillet C et al (2014) MEK in cancer and cancer therapy. Pharmacol Ther 141(2):160-171

32. Milde-Langosch K et al (2005) Expression and prognostic relevance of activated extracellular-regulated kinases (ERK1/2) in breast cancer. Br J Cancer 92(12):2206-2215

33. Hsu YL et al (2005) Asiatic acid, a triterpene, induces apoptosis and cell cycle arrest through activation of extracellular signalregulated kinase and p38 mitogen-activated protein kinase pathways in human breast cancer cells. J Pharmacol Exp Ther 313(1):333-344

34. Mamay CL et al (2003) An inhibitory function for JNK in the regulation of IGF-I signaling in breast cancer. Oncogene 22(4):602-614

35. Altiok N, Koyuturk M, Altiok S (2007) JNK pathway regulates estradiol-induced apoptosis in hormone-dependent human breast cancer cells. Breast Cancer Res Treat 105(3):247-254
36. Yu JH et al (2008) Protein tyrosine kinase, JNK, and ERK involvement in pseudolaric acid B-induced apoptosis of human breast cancer MCF-7 cells. Acta Pharmacol Sin 29(9):1069-1076

37. Cocolakis E et al (2001) The p38 MAPK pathway is required for cell growth inhibition of human breast cancer cells in response to activin. J Biol Chem 276(21):18430-18436

38. Kamaraju AK, Roberts AB (2005) Role of Rho/ROCK and p38 MAP kinase pathways in transforming growth factor-beta-mediated Smad-dependent growth inhibition of human breast carcinoma cells in vivo. J Biol Chem 280(2):1024-1036

39. Fister $\mathrm{S}$ et al (2009) GnRH-II antagonists induce apoptosis in human endometrial, ovarian, and breast cancer cells via activation of stress-induced MAPKs p38 and JNK and proapoptotic protein Bax. Cancer Res 69(16):6473-6481

40. Reshkin SJ et al (2003) Paclitaxel induces apoptosis via protein kinase A- and p38 mitogen-activated protein-dependent inhibition of the $\mathrm{Na}+\mathrm{H}+$ exchanger (NHE) NHE isoform 1 in human breast cancer cells. Clin Cancer Res 9(6):2366-2373

41. Bhoumik A, Ronai Z (2008) ATF2: A transcription factor that elicits oncogenic or tumor suppressor activities. Cell Cycle 7(15):2341-2345

42. Maekawa T et al (2008) ATF-2 controls transcription of Maspin and GADD45 alpha genes independently from p53 to suppress mammary tumors. Oncogene 27(8):1045-1054

43. Rudraraju B et al (2014) Phosphorylation of activating transcription factor-2 (ATF-2) within the activation domain is a key determinant of sensitivity to tamoxifen in breast cancer. Breast Cancer Res Treat 147(2):295-309

44. Knippen $\mathrm{S}$ et al (2009) Expression and prognostic value of activating transcription factor 2 (ATF2) and its phosphorylated form in mammary carcinomas. Anticancer Res 29(1):183-189

45. Busch $\mathrm{S}$ et al (2012) Low ERK phosphorylation in cancer-associated fibroblasts is associated with tamoxifen resistance in premenopausal breast cancer. PLoS One 7(9):e45669

46. Oh AS et al (2001) Hyperactivation of MAPK induces loss of ERalpha expression in breast cancer cells. Mol Endocrinol 15(8):1344-1359

47. Ostrakhovitch EA, Cherian MG (2005) Inhibition of extracellular signal regulated kinase (ERK) leads to apoptosis inducing factor (AIF) mediated apoptosis in epithelial breast cancer cells: the lack of effect of ERK in p53 mediated copper induced apoptosis. J Cell Biochem 95(6):1120-1134

48. Chen L et al (2009) Inhibition of the p38 kinase suppresses the proliferation of human ER-negative breast cancer cells. Cancer Res 69(23):8853-8861

49. Creighton CJ et al (2006) Activation of mitogen-activated protein kinase in estrogen receptor alpha-positive breast cancer cells in vitro induces an in vivo molecular phenotype of estrogen receptor alpha-negative human breast tumors. Cancer Res 66(7):3903-3911

50. Atanaskova $\mathrm{N}$ et al (2002) MAP kinase/estrogen receptor crosstalk enhances estrogen-mediated signaling and tumor growth but does not confer tamoxifen resistance. Oncogene 21(25):4000-4008

51. Cagnol S, Chambard JC (2010) ERK and cell death: mechanisms of ERK-induced cell death-apoptosis, autophagy and senescence. FEBS J 277(1):2-21

52. Subramaniam $S$, Unsicker K (2010) ERK and cell death: eRK1/2 in neuronal death. FEBS J 277(1):22-29

53. Mebratu Y, Tesfaigzi Y (2009) How ERK1/2 activation controls cell proliferation and cell death: is subcellular localization the answer? Cell Cycle 8(8):1168-1175

54. Courtois-Cox S et al (2006) A negative feedback signaling network underlies oncogene-induced senescence. Cancer Cell 10(6):459-472 